\title{
Disentangling neocortical alpha/beta and hippocampal theta/gamma oscillations in human episodic memory formation
}

\author{
Benjamin J. Griffiths s,b,c,*, María Carmen Martín-Buro ${ }^{\mathrm{d}}$, Bernhard P. Staresina ${ }^{\mathrm{b}, \mathrm{c}, \mathrm{e}}$, \\ Simon Hanslmayr ${ }^{b, c, f, *}$
}

${ }^{a}$ Department of Psychology, Ludwig-Maximilians-University, Munich, Germany

${ }^{\mathrm{b}}$ School of Psychology, University of Birmingham, UK

${ }^{\mathrm{c}}$ Centre for Human Brain Health, University of Birmingham, UK

${ }^{\mathrm{d}}$ Department of Psychology, Rey Juan Carlos University, Alcorcón, Madrid 28922, Spain

e Department of Experimental Psychology, University of Oxford, UK

${ }^{\mathrm{f}}$ Institute for Neuroscience and Psychology, University of Glasgow, UK

\section{A B S T R A C T}

To form an episodic memory, we must first process a vast amount of sensory information about the to-be-encoded event and then bind these sensory representations together to form a coherent memory trace. While these two cognitive capabilities are thought to have two distinct neural origins, with neocortical alpha/beta oscillations supporting information representation and hippocampal theta-gamma phase-amplitude coupling supporting mnemonic binding, evidence for a dissociation between these two neural markers is conspicuously absent. To address this, seventeen human participants completed an associative memory task that first involved processing information about three sequentially-presented stimuli, and then binding these stimuli together into a coherent memory trace, all the while undergoing MEG recordings. We found that decreases in neocortical alpha/beta power during sequence perception, but not mnemonic binding, correlated with enhanced memory performance. Hippocampal theta/gamma phase-amplitude coupling, however, showed the opposite pattern; increases during mnemonic binding (but not sequence perception) correlated with enhanced memory performance. These results demonstrate that memory-related decreases in neocortical alpha/beta power and memory-related increases in hippocampal theta/gamma phase-amplitude coupling arise at distinct stages of the memory formation process. We speculate that this temporal dissociation reflects a functional dissociation in which neocortical alpha/beta oscillations could support the processing of incoming information relevant to the memory, while hippocampal theta-gamma phase-amplitude coupling could support the binding of this information into a coherent memory trace.

\section{Introduction}

An episodic memory is a personal detail-rich, long-term memory that is anchored to a unique point in time and space (Tulving, 2002). The formation of these memories are thought to rely on both neocortical alpha/beta and hippocampal theta/gamma oscillations (Hanslmayr et al., 2016), both of which are prevalent in a wide range of human episodic memory tasks (for reviews, see Hanslmayr and Staudigl 2014; Nyhus and Curran 2010).

Neocortical alpha/beta desynchrony is thought to be beneficial for information representation (Hanslmayr et al., 2012). This idea is derived from the tenets of information theory, which propose that unpredictable states (e.g., a desynchronised network, where the firing of one neuron cannot predict the firing of another) convey substantially more information than predictable states. In direct support of this idea, neocortical alpha/beta power decreases (a proxy for neural desynchrony; Buzsáki et al., 2012; Murthy and Fetz, 1996) have been shown to correlate with the enhanced fidelity of neural representations present in BOLD signal (Griffiths et al., 2019a). Moreover, interfering with these power decreases via transcranial magnetic brain stimulation impairs episodic memory formation (Hanslmayr et al., 2014). Together, these findings (see also Fellner et al. 2013, Griffiths et al. 2021, Karlsson et al. 2020, Long and Kahana 2015, Martín-Buro et al. 2020, Sederberg et al. 2007) suggest that alpha/beta power decreases are intimately tied to the successful representation of information pertaining to episodic memories.

Hippocampal theta and gamma oscillations also play a pivotal role in episodic memory formation (Bahramisharif et al., 2018; Heusser et al., 2016; Staudigl and Hanslmayr, 2013; Tort et al., 2009). The phase of theta is thought to determine whether long-term potentiation (LTP) or long-term depression (LTD) occurs (Hasselmo et al., 2002), and gamma synchronisation compliments this process by driving neurons to fire at the frequency optimal for spike-timing dependent plasticity (STDP; Bi and Poo, 1998; Jutras et al., 2009; Nyhus and Curran, 2010). By combining these two phenomena, hippocampal theta-gamma phaseamplitude coupling is well-suited for mnemonically binding disparate

\footnotetext{
* Corresponding authors at: Department of Psychology, Ludwig-Maximilians-University, Munich, Germany and Institute for Neuroscience and Psychology, University of Glasgow, UK.

E-mail addresses: benjamin.griffiths@psy.lmu.de (B.J. Griffiths), simon.hanslmayr@glasgow.ac.uk (S. Hanslmayr).
} 
sources of information into a coherent memory trace (Griffiths and Fuentemilla, 2019; Hanslmayr et al., 2016; Lisman and Jensen, 2013).

In conjunction, neocortical alpha/beta desynchrony and hippocampal theta/gamma synchrony are thought to provide the optimal conditions for episodic memory formation (Hanslmayr et al., 2016). Tobe-encoded information is first represented in the neocortex and supported by neural desynchrony (Griffiths et al., 2019a). These representations are then passed to the hippocampus, undergoing compression so that they are supported by only a small handful of hippocampal cell assemblies (Quiroga et al., 2005). In this compressed state, these representations can then be readily associated with other elements of the ongoing episode via STDP (which benefits from neural synchrony; $\mathrm{Bi}$ \& Poo, 1998; Nyhus and Curran, 2010). Ultimately, the holistic process reflects an oscillatory division of labour in which neocortical alpha/beta desynchrony first supports the representation of relevant information in the cortex, before hippocampal theta/gamma synchrony takes precedence and binds concepts together (Griffiths et al., 2019b; Hanslmayr et al., 2016; Parish et al., 2018).

On a cognitive level however, many paradigms probing human episodic memory formation involve substantial overlap in information representation and mnemonic binding, making it difficult to conclude that their associated neural phenomena are truly dissociable. Here, we addressed this problem by using a paradigm that invokes a temporal shift in the ratio of these cognitive processes. Seventeen participants were briefly presented with a sequence of three stimuli (always consisting of an object, a feature and a scene), and then given a small window to intentionally bind these stimuli together for a later associative memory test*. We hypothesised that memory-related changes in neocortical alpha/beta activity would show a distinct temporal dynamic to memory-related changes in hippocampal theta/gamma activity, where (1) memory-related neocortical alpha/beta power decreases would be most prevalent during the perception of the sequence (from here on termed "sequence perception"), as this requires extensive processing of the details of each item prior to binding, and (2) memoryrelated increases in hippocampal theta-gamma phase-amplitude coupling would be most prevalent when participants intentionally associate the stimuli together (from here on termed "mnemonic binding"), given that theta-gamma coupling is a proxy for forms of long-term potentiation. Indeed, the results reported below support these hypotheses, suggesting that neocortical alpha/beta desynchrony and hippocampal theta/gamma synchrony arise at distinct stages of the memory formation process.

\section{Materials and methods}

\subsection{Participants}

Twenty-eight participants were recruited (mean age $=25.4$; age range $=20-33 ; 68 \%$ female; $82 \%$ right-handed). These participants received course credit or financial reimbursement in return for their participation. One participant was excluded for excessive head movement (greater than 2 standard deviations above group mean). Four participants were excluded for poor data quality (more than $50 \%$ of tri-

* While it is impossible to conclude with absolute certainty that perception and mnemonic binding are completely separable in any memory task, here we can conclude that there is a substantial shift in the ratio of the two processes. Stimulus perception will only be taking place while there is stimulus to perceive (i.e., during the presentation of the sequence), while mnemonic binding will be most prevalent when all sequence items have been presented and processed (i.e., after the final stimulus has been processed by the cortex). While this leaves room for some binding to occur towards the very end of the presentation of the last stimulus, this would be minimal in comparison to what follows during the "binding window" (see figure 1a, outlined in red). Direct contrasts of the MEG signals between sequence perception and mnemonic binding will empirically test this hypothesised shift in ratio. als rejected for artifacts). Six participants were excluded for extreme memory performance (fewer than 15 trials in one of the three memory conditions). This left seventeen participants for further analysis (mean age $=24.9$; age range $=20-32 ; 65 \%$ female; $82 \%$ right-handed). Ethical approval was granted by the Research Ethics Committee at the University of Birmingham (ERN_15-0335), complying with the Declaration of Helsinki.

\subsection{Experimental design}

Each participant completed a visual associative memory task (see Fig. 1a). During encoding, participants were presented with a line drawing of an object, a pattern, and a scene (each for $1500 \mathrm{~ms}$, with a jittered $600 \mathrm{~ms}( \pm 100 \mathrm{~ms}$ ) fixation cross shown between each stimulus). Participants were then given a short interval $(3000 \mathrm{~ms})$ to create a mental image incorporating the three stimuli to help them recall the stimuli for a later memory test. Specifically, during this window, we explicitly asked participants to create a unique fusion of the three stimuli, and suggested that they may benefit from creating bizarre or unusual mental images. We gave participants the freedom to integrate the stimuli as they saw fit, but gave the suggestion that the pattern could be blended with the object line-drawing, and the object could be placed in the scene (taking Fig. 1a as an example: participants may imagine a spotted blue-and-orange giraffe taking the tram to work). Participants were then asked to rate how difficult they found the task of associating the triad. This question was used to keep participants attending to the task, rather than provide a meaningful metric for analysis. The next trial began after the participant had responded to the difficulty question. After associating 48 triads, participants started the distractor task. In the distractor task, participants attended to a fixation cross in the centre of a black screen. The fixation cross would flash momentarily $(\sim 100 \mathrm{~ms})$ from light grey to either white or dark grey approximately every $20 \mathrm{~s}$. The participants were instructed to count the number of times the fixation cross changed to white (ignoring the times it turned dark grey) and report this value at the end of the task (approximately $2.5 \mathrm{~min}$ later). The retrieval task followed the distractor. Here, participants were presented with the line drawing (for $3000 \mathrm{~ms}$ ) and asked to recall the mental image they made during the encoding phase. Then, participants were presented with three patterns (one correct and two lures; lures were other stimuli of the same type, shown in the same block, to ensure we tested associative, rather than recognition, memory) and asked to identify the pattern associated with the line drawing. After responding, participants were presented with three scenes (one correct and two lures from the same block) and again asked to identify the pattern associated with the line drawing. After responding, participants were then asked to indicate how confident they were about their choices. They could select 'guess' (i.e., they guessed their choice), 'unsure' (i.e. they could not remember the item, but had a feeling it was the correct choice), or 'certain' (i.e. they could vividly remember the item). Participants were asked to recall all 48 triads learnt in the earlier encoding phase.

Given that participants were tasked with creating unique memories on each trial, all of which had a strong spatial component (i.e., the scene stimulus) and a unique timestamp (i.e., the position of the trial in the block), the memories formed in this experiment fit the classic description of an episodic memory.

Participants completed four blocks of this task (192 trials in total). The order in which the pattern and scene were presented during perception was swapped between each block (where a "block" is defined as a complete cycle of encoding, distractor and retrieval tasks). On blocks where scenes preceded patterns during perception, the presentation order at retrieval was also reversed.

For all responses, participants used two non-magnetic, single-finger optical response pads. The left pad allowed participants to cycle through the possible responses, and the right pad allowed participants to confirm their selection. 
(a)

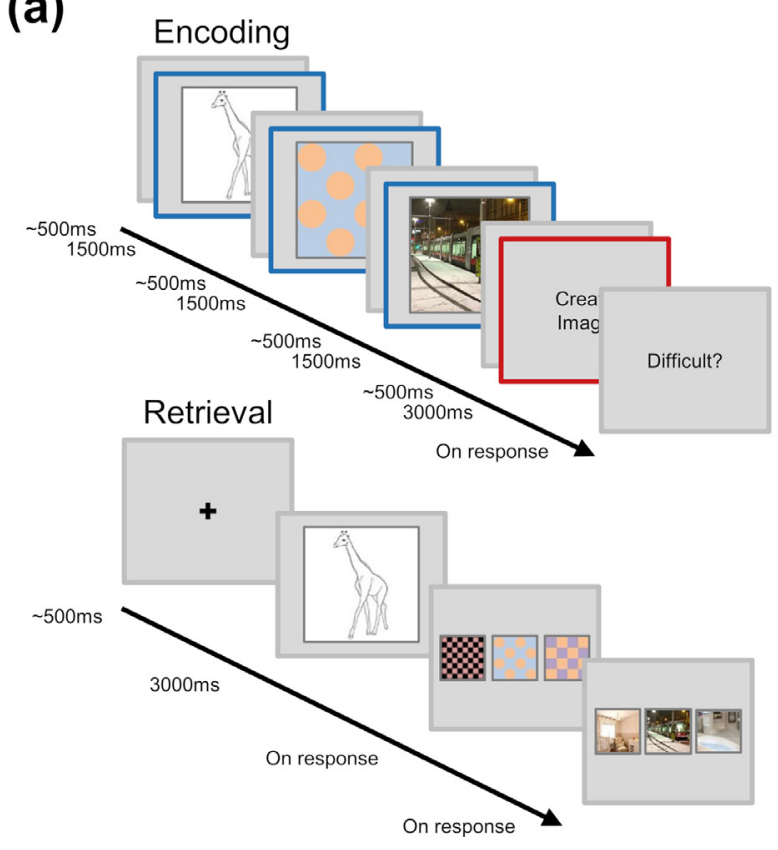

(b)

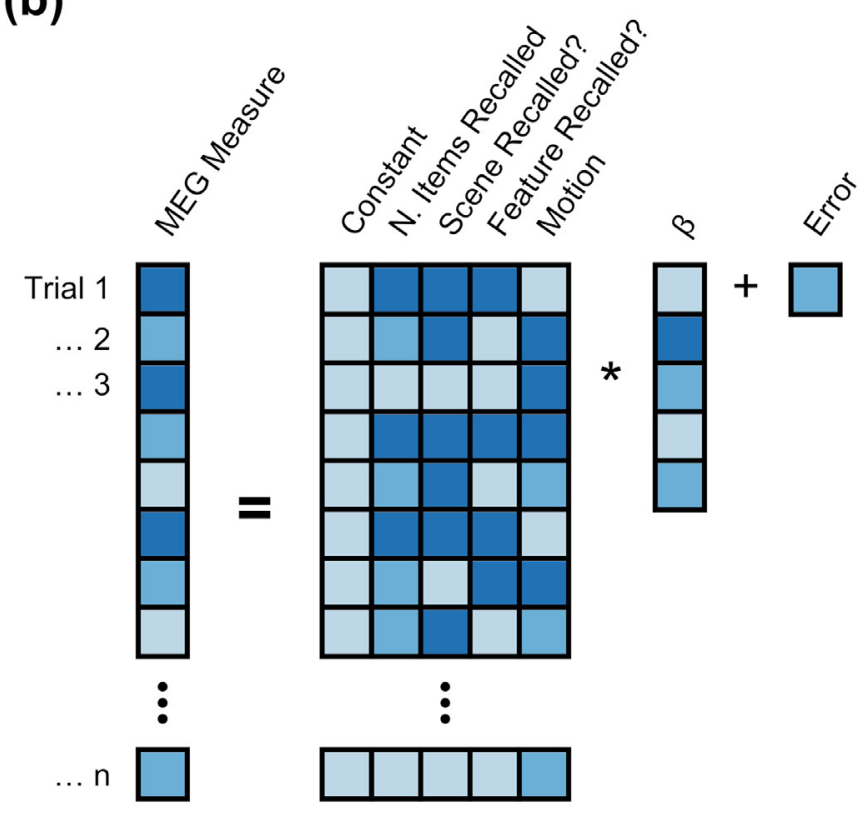

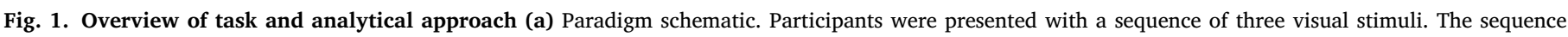

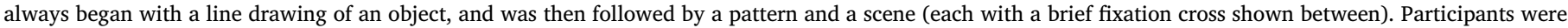

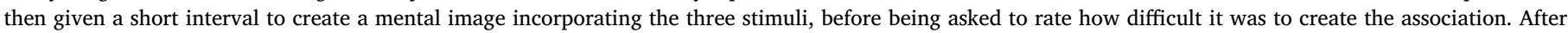

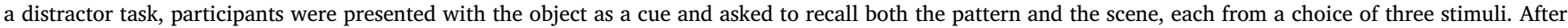

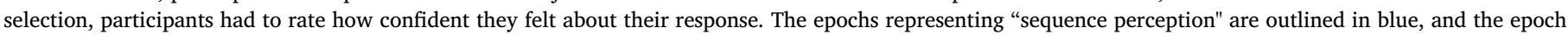

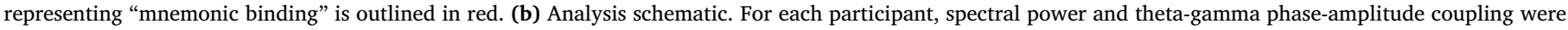

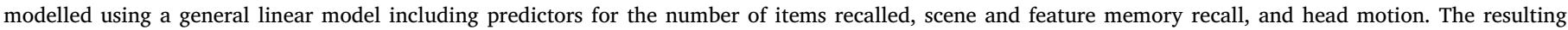

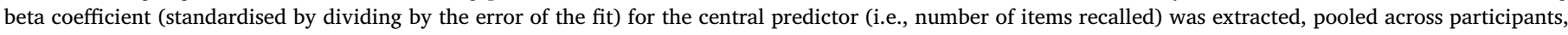

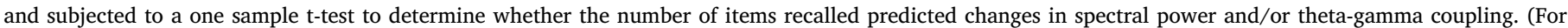
interpretation of the references to color in this figure legend, the reader is referred to the web version of this article.)

\subsection{Behavioural analysis}

For each trial, memory performance was coded as either 'complete' (i.e., they remembered both the scene and the pattern), 'partial' (i.e. they remembered only one of the associates), or 'forgotten' (i.e. they remembered neither the scene nor the pattern). Any selection where the participant indicated that they guessed was marked as a 'miss'.

\subsection{MEG acquisition}

MEG data was recorded using a 306-channel (204 gradiometers, 102 magnetometers) whole brain Elekta Neuromag TRIUX system (Elekta, Stockholm, Sweden) in a magnetically shielded room. Participants were placed in the supine position for the duration of the experiment. Data was continuously recorded at a sampling rate of $1000 \mathrm{~Hz}$. The head shape of each participant (including nasion and left/right ear canal) was digitised prior to commencing the experiment. Continuous head position indicators (cHPI) were recorded throughout. The frequencies emitted by the cHPI coils were $293 \mathrm{~Hz}, 307 \mathrm{~Hz}, 314 \mathrm{~Hz}$ and $321 \mathrm{~Hz}$. Magnetometer data was excluded from the main analysis as they contained substantial noise that could not be effectively removed or attenuated.

\section{5. $M E G$ preprocessing}

All data analysis was conducted in Matlab using Fieldtrip (Oostenveld et al., 2011) in conjunction with custom scripts. First, the data was lowpass filtered at $165 \mathrm{~Hz}$ to remove the signal generated by the HPI coils. Second, the data was epoched around each event of interest. At encoding, the epochs reflected the time windows where each stimulus was presented (from here on termed 'sequence perception') and when the 'associate' prompt was presented (termed 'mnemonic binding'). Sequence perception epochs began $2000 \mathrm{~ms}$ before stimulus onset and ended $3500 \mathrm{~ms}$ after onset (that is, $2000 \mathrm{~ms}$ after stimulus offset [total epoch duration: $5500 \mathrm{~ms}$ ]). Mnemonic binding epochs began $2000 \mathrm{~ms}$ before stimulus onset and ended $5000 \mathrm{~ms}$ after onset (that is, $2000 \mathrm{~ms}$ after stimulus offset [total epoch duration: 7000ms]). Note that the length of these epochs extended beyond the phase of interest to account for potential filter artifacts, particularly for the case of our cross-frequency coupling analyses, but were subsequently restricted to phase of interest when conducting statistical analysis. Third, independent components analysis was conducted, and any identifiable eye-blink or cardiac components were removed. Fourth, the data was visually inspected, and any artefactual epochs or sensors were removed from the dataset (mean percentage of trials removed: $18.0 \%$; range: $5.7-32.2 \%$ ).

\subsection{Movement correction}

To identify participants with extreme head motion during MEG recordings, the recorded data was first highpass filtered to $250 \mathrm{~Hz}$ to isolate the cHPI signal. Second, the variance of the signal for each sensor was computed across every time point of the continuous recording. Third, the variance was mean averaged across sensors to provide a singular estimate of change in cHPI signal across the duration of the experiment. Fourth, the mean variance and its standard deviation was calculated across participants. Lastly, participants with extreme head motion were identified as those with variance greater than two standard deviations above the group mean. These participants were excluded from further analysis.

To help attenuate motion-related confounds in the spectral power and phase-amplitude coupling analyses, a trial-by-trial estimate of mo- 
tion was calculated. First, the data was highpass filtered at $250 \mathrm{~Hz}$. Second, the data was epoched into trials matching those outlined in the section above. Third, the envelope of the signal in each epoch was calculated (to avoid issues of mean phase angle difference in cHPI signal across trials). Fourth, the envelope was averaged over time to provide a single value for each epoch and channel. Fifth, the dot product was computed across sensors between the first epoch and every other epoch (algebraically: $\sum_{i=1}^{n} a_{i} b_{i}$, where $n$ is the number of channels, $a_{i}$ is the power at sensor $i$ during the first trial, and $b_{i}$ is the power at sensor $i$ during the trial of interest). This provided a single value (between zero and infinity) for each trial that described how similar the topography of that trial was to the first trial - the higher the value, the more similar the topographies are between the two trials (with the assumption that the more dissimilar a cHPI topography is to the starting topography, the more the head has deviated from its starting position). These values were entered as a regressor of no interest in the central multiple regression analyses.

\subsection{Time-frequency decomposition and statistical analysis}

Sensor-level time-frequency decomposition was conducted on the sequence perception and mnemonic binding epochs. For low frequencies, the preprocessed data was first convolved with a 6-cycle wavelet $(-0.5$ to $3 \mathrm{~s}$ [to $2 \mathrm{~s}$ for perceptual epochs to avoid the subsequent stimulus], in steps of $50 \mathrm{~ms} ; 2-40 \mathrm{~Hz}$; in steps of $1 \mathrm{~Hz}$ ). For high frequencies, Slepian multitapers were first used to estimate power $(-0.5$ to $3 \mathrm{~s}$ [to $2 \mathrm{~s}$ for perceptual epochs], in steps of $50 \mathrm{~ms} ; 40$ to $100 \mathrm{~Hz}$, in steps of $4 \mathrm{~Hz}$ ). For this latter analysis, frequency smoothing was set to one quarter of the frequency of interest and temporal smoothing was set to $200 \mathrm{~ms}$. Second, planar gradiometers were combined by summing the power of the vertical and horizontal components. Third, for perceptual trials only, power was then averaged over the three stimulus presentation windows of each triad to provide mean power during perception of the triad. Any triads where one or more epochs had been rejected during preprocessing were excluded at this stage. We averaged spectral power across the three windows as we reasoned that this approach would be most sensitive to changes in spectral power that predicted the number of items later recalled. To successfully recall a stimulus, we assume that an alpha/beta power decrease must arise in two of the windows - the initial line drawing (i.e., the retrieval cue) and the to-be-recalled stimulus. As such, focusing analyses on a single stimulus is less sensitive to later memory performance than an aggregate measure created by averaging across the epochs. Fourth, the background $1 / \mathrm{f}$ characteristic was subtracted using an iterative linear fitting procedure.

To isolate oscillatory contributions, $1 / \mathrm{f}$ activity was attenuated in the time-frequency data by subtracting the linear fit of the $1 / \mathrm{f}$ characteristic (Griffiths et al., 2019b; Manning et al., 2009; Zhang and Jacobs, 2015). To this end, a vector containing values of each derived frequency $(A)$ and another vector containing the power spectrum, averaged over all time-points and trials of the relevant memory condition (B) were log-transformed to approximate a linear function. The linear equation $A x=B$ was solved using least-squares regression, where $x$ is an unknown constant describing the $1 / \mathrm{f}$ characteristic. The $1 / \mathrm{f}$ fit $(A x)$ was then subtracted from the log-transformed power spectrum $(B)$. As this fit can be biased by outlying peaks (Haller et al., 2018), an iterative algorithm was used that removed probable peaks and then refitted the $1 /$ f. Outlying peaks in this $1 / \mathrm{f}$-subtracted power spectrum were identified using a threshold determined by the mean value of all frequencies that sat below the linear fit. The MEG power spectrum is the summation of the $1 / \mathrm{f}$ characteristic and oscillatory activity (i.e., at no point does oscillatory activity subtract from the $1 / \mathrm{f}$ ), therefore all values that sit below the linear fit can be seen an estimate error of the fit. Any peaks that exceed the threshold were removed from the general linear model, and the fitting was repeated. Notably, as power for the low frequencies $(2-40 \mathrm{~Hz})$ and high frequencies $(40-100 \mathrm{~Hz})$ was calculated using different methods (wavelets and Slepian multitapers, respectively), the two bands have disparate levels of temporal and spectral smoothing. To avoid a spurious fitting due of the $1 / \mathrm{f}$ because of these differences, the $1 / \mathrm{f}$ correction was conducted separately for these two bands.

For statistical analysis, a trial-based multiple regression was run for each participant. Four regressors were used to predict observed power for every channel $\mathrm{x}$ frequency $\mathrm{x}$ time point independently. These four regressors were (1) number of items recalled, (2) whether the scene was recalled, (3) whether the pattern was recalled, (4) the change in head position [based on the motion calculation outlined above]. The first regressor was of primary interest, the second and third regressors isolated spectral power changes that are unique to scene-specific and patternspecific encoding processes (respectively), and the fourth regressor accounted for changes in spectral power driven by head movement (see the next paragraph for notes on multicollinearity). The beta weight of the first regressor, obtained for a given channel $\mathrm{x}$ frequency $\mathrm{x}$ time point, was then standardised by dividing the standard error of the fit (providing a $t$-value) to attenuate the impact of poor model fits on the final analysis. Here, a positive $t$-value would indicate that spectral power increases with more items recalled, and a negative beta coefficient would indicate that spectral power decreases with more items recalled. The beta coefficients for each participant were pooled across the sample and entered into a one-tailed cluster-based permutation test (focused upon $8-30 \mathrm{~Hz}$ post-stimulus [0-1500ms] activity; 2000 permutations, alpha threshold $=0.05$, cluster alpha threshold $=0.05$, minimum neighbourhood size $=3$; (Maris and Oostenveld, 2007) to examine whether the observed fits consistently deviated from the null hypothesis $(t=0)$ across participants. Clusters that produced a p-value less than 0.05 were considered significant. Cohen's $d_{z}$ was used as the measure of effect size for these clusters (Lakens, 2013), where $d_{z}=\frac{\bar{t}}{\sqrt{n}}, \bar{t}=$ mean t-statistic within the cluster, $n=$ number of participants.

Following the visualisation of spectral power during the mnemonic binding window, we observed an unexpected increase in low-frequency power correlating with enhanced memory performance. To statistically appraise this, we used the same statistical procedure as above with a single alteration: the use of two-tailed statistical tests, which reflects our lack of directional hypotheses in this analysis.

Notably, it is plausible to suggest that the three memory regressors are, to some extent, correlated and that this would introduce multicollinearity into the regression models. To test this, we calculated the Variance Inflation Factor (VIF)-a measure of the magnitude of multicollinearity. Rule of thumb suggests that a VIF greater than 10 is considered high and could compromise the model (Kutner et al., 2004). The VIF between number of items recalled and scene recall success was, on average, 2.615 (s.d. 2.621), and the VIF between number of items recalled and pattern recall success was, on average, 1.027 (s.d. 0.033). As these values fall below the threshold of 10 , multicollinearity is not an apparent concern.

An additional analysis was conducted to confirm that alpha/beta power did indeed decrease following the onset of the sequence stimuli. Here, spectral power was computed as described in the first paragraph of this sub-section ('Time-frequency decomposition and statistical analysis'). Power was then averaged across trials and baseline-corrected via z-transformation. To this end, for each channel and frequency, the mean and standard deviation of pre-stimulus power $(-500 \mathrm{~ms}$ to stimulus onset) across time was computed, and then for every time point, spectral power was normalised by subtracting the pre-stimulus mean, and then dividing by the pre-stimulus standard deviation. This returned a power spectrum in which any negative value indicates that power dropped relative to baseline. The time-frequency spectra for each participant were pooled across the sample and entered into a one-tailed cluster-based permutation test (2000 permutations, alpha threshold $=0.05$, cluster alpha threshold $=0.05$, minimum neighbourhood size $=3$; Maris and Oostenveld, 2007) to examine whether post-stimulus power decreased (i.e., $z<0$ ) relative to baseline beyond what might be expected by chance. 


\subsection{Source analysis}

The preprocessed data was reconstructed in source space using individual head models and structural (T1-weighted) MRI scans for all but two individuals who did not wish to return for an MRI scan. For these two individuals, a standard head model and MRI scan was used (taken from the Fieldtrip toolbox; for details, see http://www.fieldtriptoolbox.org/template/headmodel). The head shape (together with the HPI coil positions) of each participant was digitised using a Polhemus Fasttrack system. The timelocked MEG data was reconstructed using a single-shell forward model and a Linearly Constrained Minimum Variance beamformer (LCMV; van Veen et al., 1997). The lambda regularisation parameter was set to $1 \%$. The source model consisted of virtual electrodes spaced $10 \mathrm{~mm}$ apart in all planes, and covered the entire brain.

\subsection{MEG phase-amplitude coupling computation and statistical analysis}

For the phase-amplitude coupling analyses, we focused our analysis directly on source-reconstructed hippocampal virtual sensors. Given the depth and size of the hippocampus (it makes up around $1 \%$ of the MEG sourcemodel), it makes most sense to move directly to source space and analyse source-localised measures of hippocampal activity.

To calculate the extent to which hippocampal gamma activity coupled to hippocampal theta phase, the modulation index (MI) was calculated (Tort et al., 2010). First, the peak theta and gamma frequencies were calculated by estimating power across all hippocampal virtual sensors (bilaterally, as defined by the automated anatomical labelling [AAL] atlas) using the same time-frequency decomposition method reported above ${ }^{\dagger}$. The Matlab function findpeaks() was then used to extract the most prominent peak within the theta $(2-7 \mathrm{~Hz})$ and gamma $(40-80 \mathrm{~Hz})$ bands for each participant. Across participants, the mean theta peak was at $5.1 \mathrm{~Hz}$ (standard deviation: $1.0 \mathrm{~Hz}$; range: $3.1-7.0 \mathrm{~Hz}$ ), and the mean gamma peak was at $66.1 \mathrm{~Hz}$ (standard deviation: $4.6 \mathrm{~Hz}$; range: $59.0-73.0 \mathrm{~Hz}$ ) [see Supplementary Fig. 1 for all plots; see Supplementary Fig. 2 for time-series]. Second, the time-series of the hippocampal virtual sensors were duplicated, with the first being filtered around the theta peak $( \pm 0.5 \mathrm{~Hz})$ and the second being filtered around the gamma peak $( \pm 5 \mathrm{~Hz})$. Third, the Hilbert transform was applied to the theta- and gamma-filtered time-series, with the phase of the former and power of the latter being extracted. Fourth, the time-series data was re-epoched, beginning $500 \mathrm{~ms}$ after the onset of the stimulus/fixation cross and at the onset of the next screen. This attenuated the possibility that an event-related potential and/or edge artifacts from the filtering/Hilbert transform could influence the phase-amplitude coupling measure (Aru et al., 2014). Fifth, gamma power was binned into 12 equidistant bins of $30^{\circ}$, according to the concurrent theta phase. This binning was conducted for each trial and sensor separately. Sixth, the MI was computed by comparing the observed distribution to a uniform distribution. Seventh, the resulting MI values were subjected to a trialbased multiple regression conducted in the same manner as for the spectral power analyses. However, two additional regressors were added to this model: (1) hippocampal peak theta power [per trial, averaged across $500 \mathrm{~ms}$ to $3000 \mathrm{~ms}$ ], (2) hippocampal peak gamma power [per trial, averaged across $500 \mathrm{~ms}$ to $3000 \mathrm{~ms}$ ]. These regressors addressed

\footnotetext{
$\dagger$ Though the notion of localising deep regions such as the hippocampus was once controversial, an ever-growing number of studies have suggested that it is achievable. Ruzich et al. (2019) uncovered 29 studies that used gradiometers alone to localise hippocampal signals, while Dalal et al. (2013) demonstrated that MEG signals directly correlate with simultaneously recorded intracranial hippocampal recordings. As such, the theoretical notion that the hippocampus cannot be measured using MEG has been refuted by numerous empirical demonstrations to the contrary.
}

the potential confound of concurrent power influence phase estimates (Aru et al., 2014). Eighth, these results were averaged over hippocampal virtual sensors and these participant-specific t-values were subjected to a permutation-based one-sample t-test contrasting memory-related changes in phase-amplitude coupling to the null hypothesis $(t=0)$. Notably, as we had focused our analyses on the peak theta and gamma frequencies, and used the average PAC values across virtual sensors, only a single statistical comparison was made. Therefore, no cluster-based multiple comparison correction was required. As the p-values reported here are estimated by permutation rather than from the parametric test, the t-values and degrees of freedom we report should only be used for reference.

To explore how phase-amplitude coupling changed as a function of sequence position (that is, does coupling increase as the sequence progresses?), we computed the modulation index as above and then conducted a variant on the original regression model. Here, for each stimulus epoch, we modelled phase-amplitude coupling as the product of five regressors: (1) a constant, (2) the position of each item in the sequence (modelled as values ' 1 ', ' 2 ', and ' 3 '), (3) the power of the phase-giving (i.e., theta) frequency, (4) the power of the power-giving (i.e., gamma) frequency, and (5) motion. As above, our regressor of interest (here: sequence position) was averaged over hippocampal virtual sensors and the participant-specific t-values were subjected to a permutation-based one-sample $t$-test.

We examined the spatial specificity of this effect by using the same pipeline as above to assess theta-gamma phase-amplitude coupling in the frontal, occipital, parietal, and temporal lobes (individually; as defined by the wfupickatlas toolbox for SPM). This analysis (plus source visualisation) help confirm that theta-gamma coupling observed in the hippocampus ROI originated from the hippocampus itself, rather than "bled in" from another region.

To compliment this analysis, we also ran a searchlight-based analysis. Here, we aimed to contrast the magnitude of the memory-related hippocampal coupling effect with memory-related coupling effects outside the hippocampus (in searchlights including approximately the same number of voxels; assuaging concerns that the lobe-based ROIs were too broad to detect local coupling effects). To this end, we iterated through every source voxel, identified its immediate neighbours (those immediately in front of and behind the voxel in 3-dimensional space [minicluster size: 27 voxels; for comparison, hippocampal $\mathrm{ROI}=25$ voxels]), and took the mean memory-related hippocampal phase-amplitude coupling within this mini-cluster. For each mini-cluster, this mean value was then contrasted against chance, and the resulting t-statistic was added to a distribution describing the magnitude of memory-related phase-amplitude coupling across the brain. A p-value was then dervied by comparing hippocampal coupling to the whole-brain distribution (as done in a permutation test), allowing us to infer the extent to which hippocampal phase-amplitude coupling deviated from what was typical within the brain.

An additional analysis was conducted to confirm that hippocampal theta/gamma coupling did increase during the mnemonic binding window. Here, the modulation index was calculated in the same manner as described above, and then z-transformed using the mean and standard deviation of a surrogate distribution, where the gamma time-series was randomly shuffled across trials relative to the theta phase and the modulation index was re-computed (that is: the theta time-series of a given trial was randomly paired with the gamma time-series of a different trial; 50 permutations). This returned a $z$-score in which any positive value would indicate hippocampal theta-gamma coupling was greater than what would be expected by chance. The z-transformed modulation index for each participant was pooled across the sample and entered into a one-tailed permutation test (2000 permutations) to examine whether hippocampal phase-amplitude coupling exceed chance during mnemonic binding. 


\section{Results}

\subsection{Behavioural results}

Participants, on average, correctly recalled both the associated pattern and associated scene on $38.3 \%$ of trials, recalled only one associated stimulus on $34.4 \%$ of trials, and failed to recall either associate on $27.3 \%$ of trials. Participants correctly recalled the associated pattern on $49.2 \%$ of trials, and correctly recalled the associated scene on $82.1 \%$ of trials (both of which are well above chance performance [33.3\%]). A paired-samples t-test revealed that memory for scenes was substantially greater than memory for patterns $\left(p<0.001\right.$, Cohen's $\left.d_{z}=4.31\right)$. To attenuate the impact of differing memory performance for the two stimulus types in the subsequent analyses, two regressors were included in all models that served to suppress variance attributable to scene-specific and feature-specific memory.

\subsection{Neocortical alpha/beta power decreases during sequence perception predict enhanced memory performance}

After establishing that alpha/beta power did indeed decrease from baseline following the presentation of the sequence stimuli $\left(\mathrm{p}_{\text {corr }}<\right.$ 0.001 , Cohen's $d_{z}=1.22$, cluster size $=28,260$, mean t-statistic within cluster $=-5.03$; see Fig. $2 \mathrm{a}$ ), we set out to test our first hypothesis: are memory-related decreases in alpha/beta power more prevalent during the perception of the sequence than during mnemonic binding?

To this end, we estimated single-trial spectral power, and then used participant-specific regression models to estimate the extent to which spectral power changes as a function of the number of items later recalled. The resulting beta co-efficients (standardised by the standard error of the fit, resulting in participant-specific $t$-values) were then pooled across participants and entered into a cluster-based permutation test. This cluster-based analysis revealed a significant effect where a decrease in the magnitude of alpha/beta power correlated with an increase in the number of items later recalled ( $\mathrm{p}_{\text {corr }}=0.032$, Cohen's $\mathrm{d}_{\mathrm{z}}=0.60$, cluster size $=1013$, mean $t$-statistic within cluster $=-2.47$; see Fig. $2 \mathrm{~b}$ and $2 \mathrm{~d}$ ). This cluster extended over the posterior sensors, bilaterally, between 8 and 15Hz (see Fig. 2b). Source reconstruction confirmed this localisation, implicating bilateral early occipital regions (see Fig. 2e). Parsimonious results were found during retrieval (see Supplementary Fig. 3), and when breaking the memory effects down by stimulus type (see Supplementary Fig. 4).

No memory-related changes in theta power $\left(2-7 \mathrm{~Hz} ; \mathrm{p}_{\text {corr }}=0.101\right)$, "slow" gamma power $(40-60 \mathrm{~Hz}$; no cluster formed), or "fast" gamma power $(60-100 \mathrm{~Hz}$; no cluster formed) were observed during the presentation of the sequence.

No change in alpha/beta power was observed when participants were asked to engage in mnemonic binding ( $\mathrm{p}_{\text {corr }}>0.5$; see Fig. $\left.2 \mathrm{c}\right)$. Similarly, no memory-related changes in theta power $\left(2-7 \mathrm{~Hz} ; \mathrm{p}_{\text {corr }}=0.130\right)$, "slow" gamma power $(40-60 \mathrm{~Hz}$; no cluster formed), or "fast" gamma power (60-100Hz; no cluster formed) were observed when participants were asked to engage in mnemonic binding.

A direct contrast of spectral power between sequence perception and mnemonic binding demonstrated that the inverse relationship between alpha power and subsequent memory performance was significantly more pronounced during perception ( $\mathrm{p}_{\text {corr }}=0.014$, Cohen's $\mathrm{d}_{\mathrm{z}}=0.60$, cluster size $=794$, cluster t-statistic $=-2.49$; see Fig. 2 f). Together, these findings suggest that alpha/beta power decreases during sequence perception, but not during mnemonic binding, scale with the number of items that are later recalled.

\subsection{Hippocampal theta/gamma phase-amplitude coupling during mnemonic binding, but not sequence perception, predicts successful episodic memory formation}

We then probed how hippocampal theta/gamma phase-amplitude coupling relates to episodic memory formation. Here, we identified the participant-specific peaks in hippocampal theta and gamma power (see Fig. 3a) and then used the modulation index (Tort et al., 2010) to approximate theta-gamma phase amplitude coupling. In the first instance, we asked whether theta-gamma phase amplitude coupling exceeds what would be expected by chance. Indeed, during mnemonic binding, theta-gamma coupling was greater than chance for trials which participants later successfully recalled at least one associate ( 2 items recalled: $\mathrm{t}(16)=1.84, p=0.044, d=0.45 ; 1$ item recalled: $\mathrm{t}(16)=2.02$, $p=0.037, d=0.49$ ), though no effect was observed when the associated stimuli were later forgotten $(p>0.5)$. During sequence perception, theta-gamma coupling never exceeded chance in any memory condition (all items: $\mathrm{t}(16)=0.85, p=0.198, d=0.21 ; 2$ items recalled: $\mathrm{t}(16)=0.20, p=0.429, d=0.05 ; 1$ item recalled: $\mathrm{t}(16)=0.79, p=0.222$, $d=0.19$; no items recalled: $\mathrm{t}(16)=0.19, p=0.431, d=0.05$ ).

We then used the same regression-based approach used for the spectral power analyses above to quantify how theta-gamma coupling differed as a function of the number of items recalled. During mnemonic binding, increases in hippocampal theta/gamma phase-amplitude coupling scaled with the number of items later recalled $(\mathrm{t}(16)=2.24$, $p=0.020$, Cohen's $d_{z}=0.54$; see Fig. $\left.3 b-d\right)$. There was no significant memory-related change in theta-gamma coupling during sequence perception ( $p>0.5$; see Fig. 3e). This lack of change was not attributable to the fact that coupling during sequence perception was estimated using shorter epochs, as we continued to observe significant coupling during mnemonic binding when the binding epochs were shortened to match the length of the sequence perception epochs $(\mathrm{t}(16)=1.85, p=0.043$, Cohen's $d_{z}=0.45$ ). A direct contrast in PAC between sequence perception and mnemonic binding revealed that memory-related increases in PAC are more pronounced during mnemonic binding $(\mathrm{t}(16)=1.93$, $p=0.040$, Cohen's $d_{z}=0.47$; see Fig. $3 f$ ). In conjunction, these findings suggest that memory-related theta/gamma phase-amplitude coupling is restricted to periods of mnemonic binding.

To ensure that the hippocampal effect observed during mnemonic binding was not a result of spatial smearing from some other region, we re-ran this analysis using four additional regions of interest: the frontal lobe, parietal lobe, temporal lobe (excluding the hippocampus), and the occipital lobe. None of these regions exhibited significant theta-gamma phase-amplitude coupling during mnemonic binding (frontal: $p=0.308$, parietal: $p=0.250$, temporal: $p=0.078$, occipital: $p=0.169$ ). Furthermore, a searchlight-based analysis revealed that hippocampal phaseamplitude coupling was substantially greater than other searchlightbased regions-of-interest that matched the size of the hippocampus ( $p=0.024$; see Fig. $2 \mathrm{~d}$ for visualisation of source map; see Supplementary Fig. 6 for histogram of searchlight statistics). Together, these results suggest that the memory-related enhancement in hippocampal theta/gamma phase-amplitude coupling is indeed originating from the source-reconstructed hippocampus, as opposed to "bleeding in" from neighbouring regions.

Notably, theta-gamma coupling seemed to be substantially greater in the right, relative to the left, hippocampus. We are, however, hesistant to draw conclusions about this difference as LCMV beamformers often fail to separate correlated sources (e.g., left and right hippocampal signals), and hence generate illusory lateralisation effects (O'Neill et al., 2021). As such, here we simply focus on the region, rather than the hemisphere, where the effect arose.

As can be seen in Fig. 3b, there is an apparent memory-related shift in the phase at which gamma couples to theta during mnemonic binding. As the modulation index used above is insensitive to such shifts, we statistically appraised this effect using a circular-to-linear correlation. Alas, no consistent change was found $(p=0.435)$ suggesting that gamma activity does not precess along the phase of theta as a function of the number of items later recalled.

While these results all support the idea that theta-gamma coupling is specific to mnemonic binding, a question remains as to why, on a neurophysiological level, theta/gamma phase-amplitude coupling was more prevalent during mnemonic binding relative to sequence percep- 
(a) Spectral Power during Sequence Presentation (across all trials)

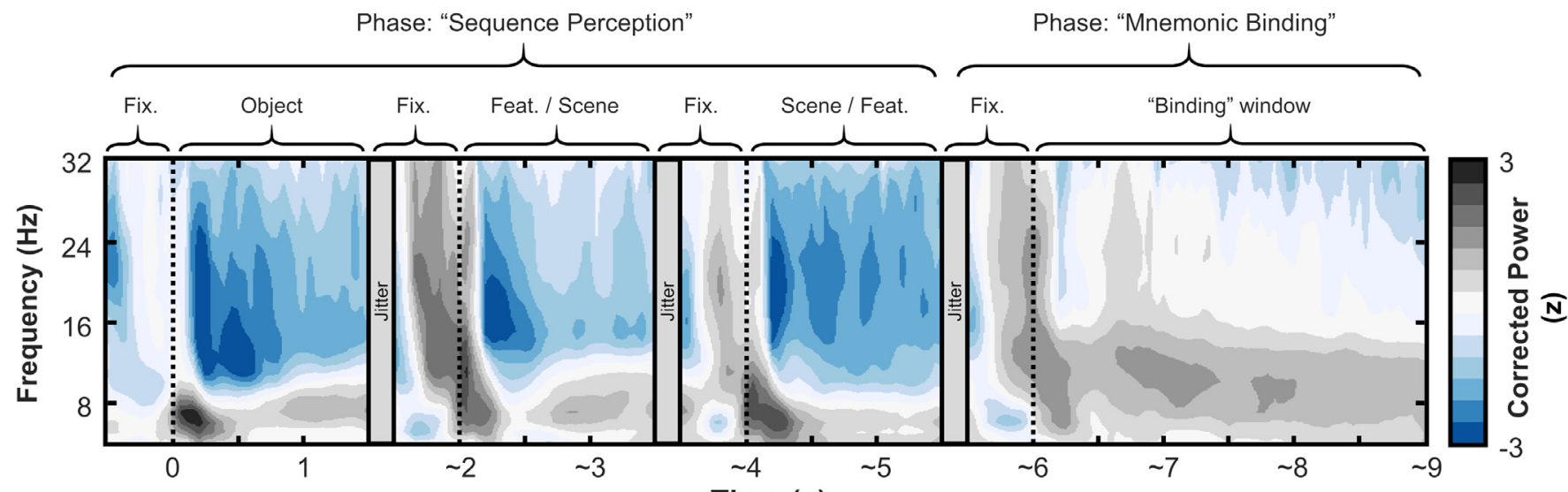

(b)

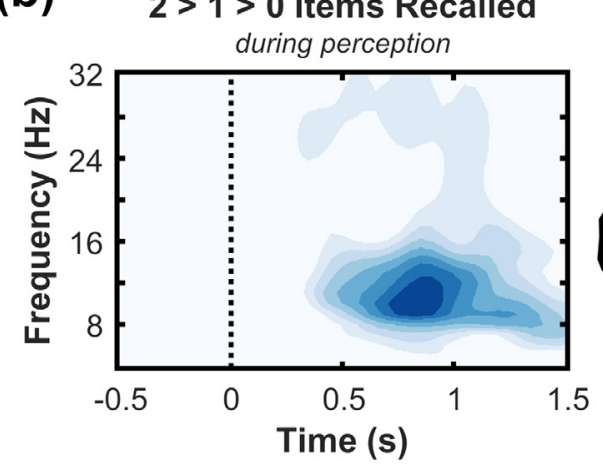

(d)

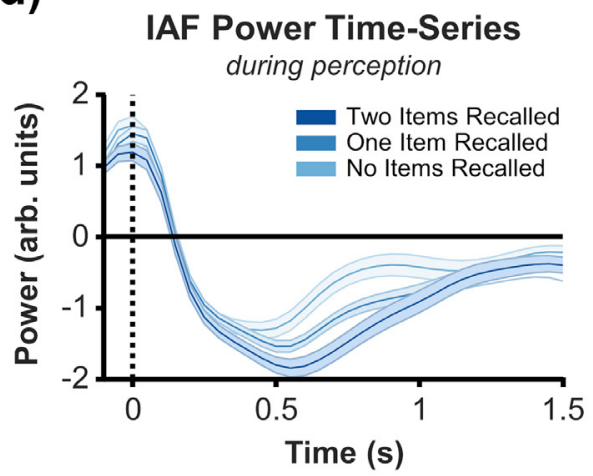

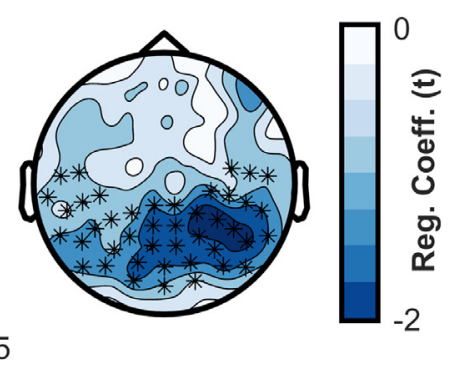

(e)

$2>1>0$ Items Recalled alpha power during perception

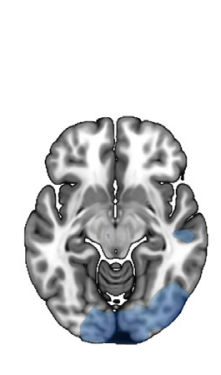

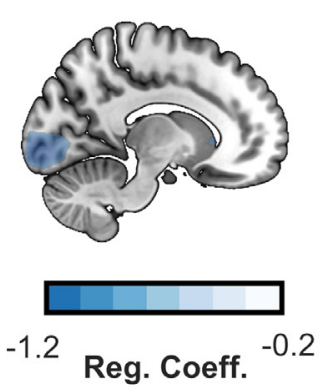

(t) (c)

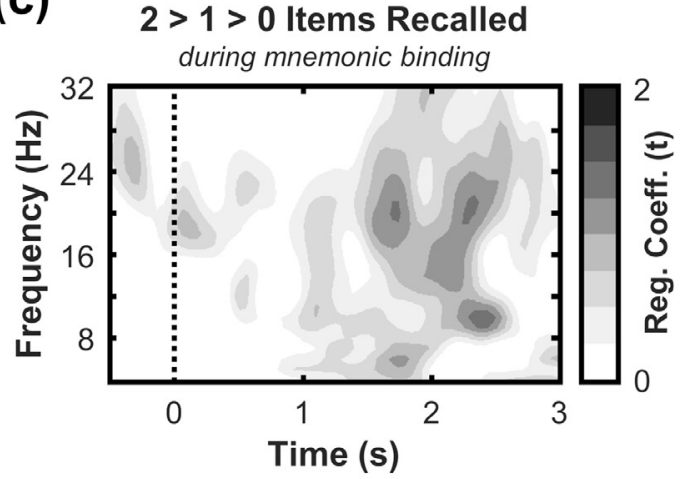

(f)

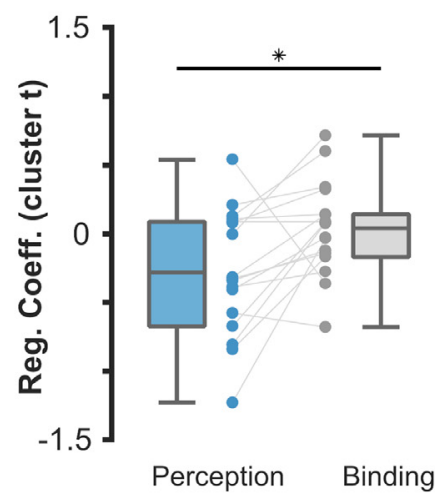

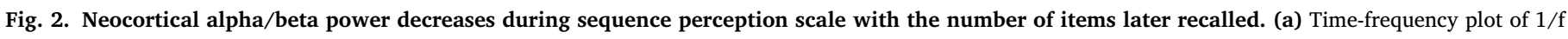

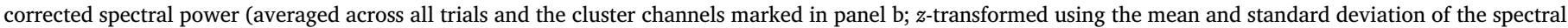

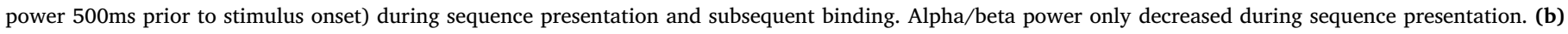

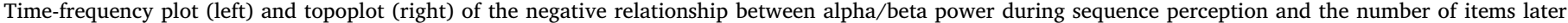

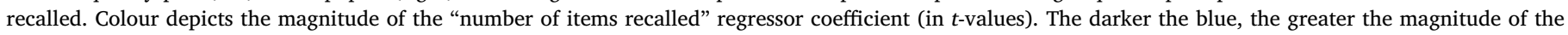

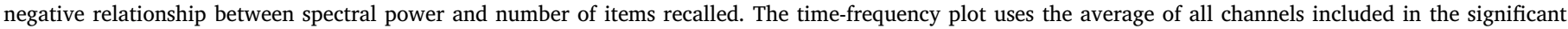

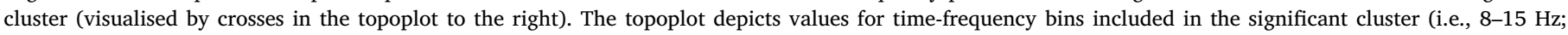

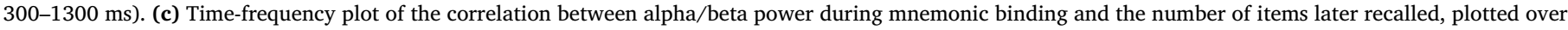

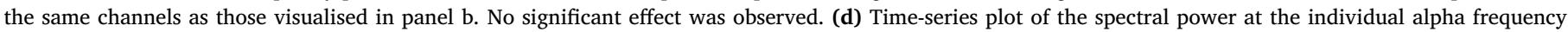

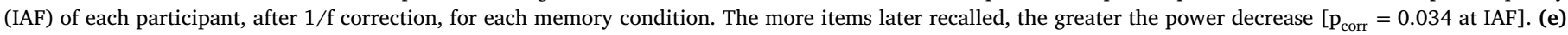

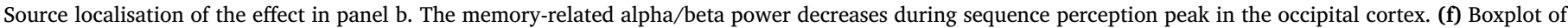

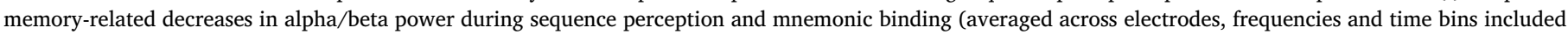

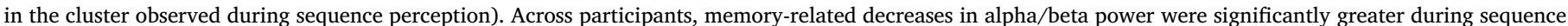

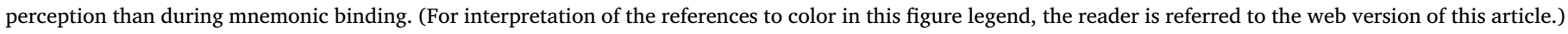




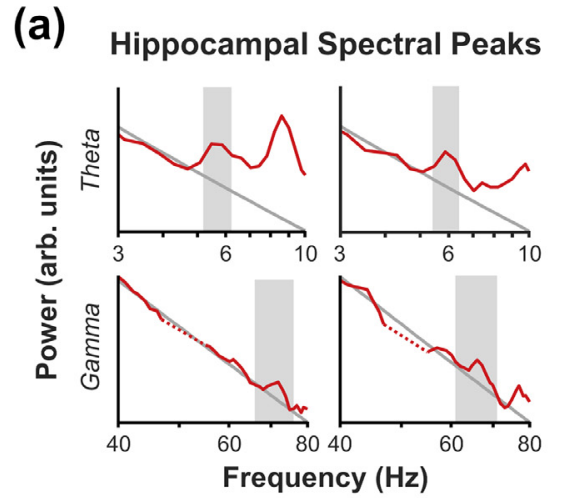

(d) $2>1>0$ Items Recalled
$P A C$ during mnemonic binding
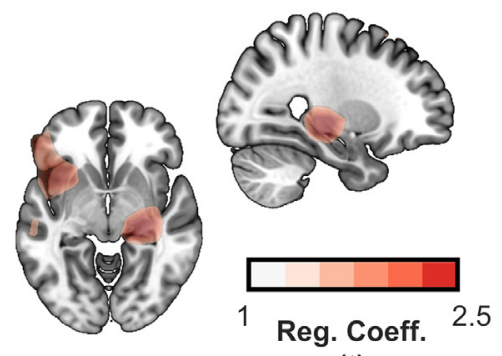

(t) (b) Hipp. Theta-Gamma Coupling during mnemonic binding

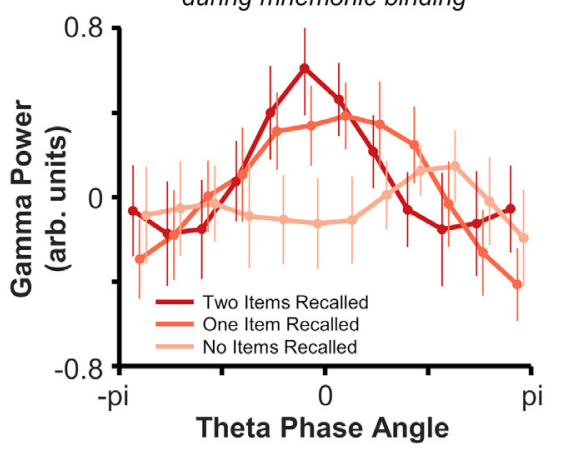

(e)

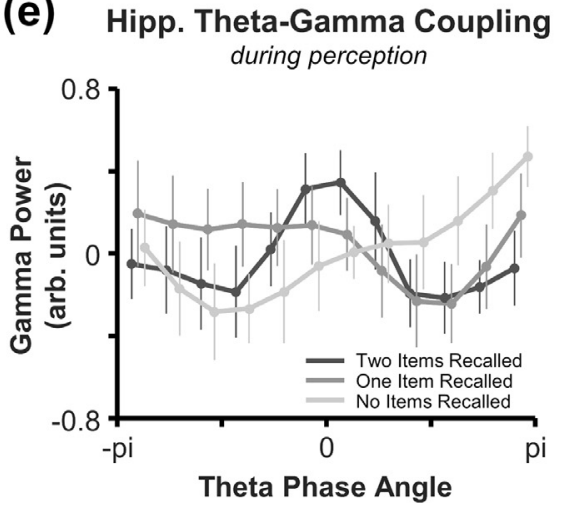

(C) $2>1>0$ Items Recalled

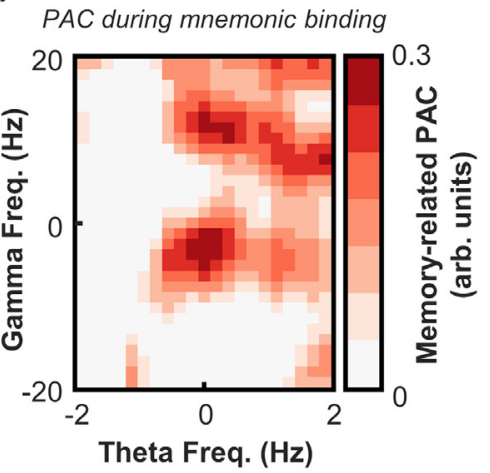

(f)

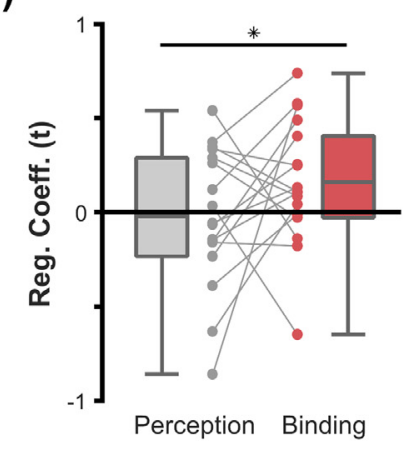

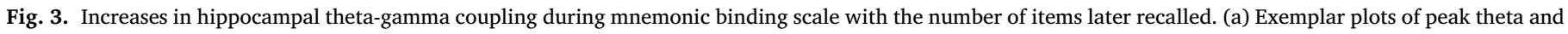

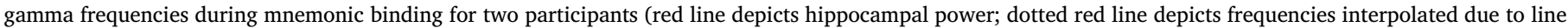

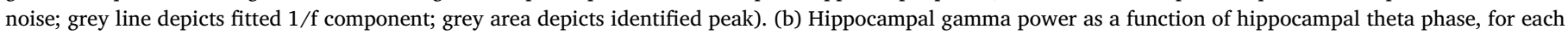

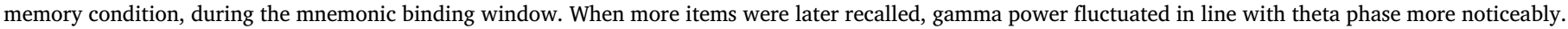

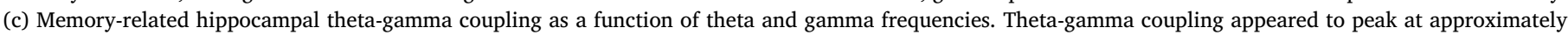

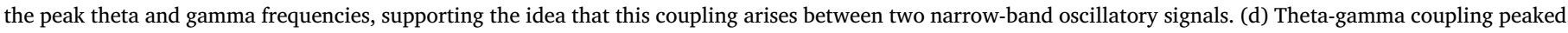

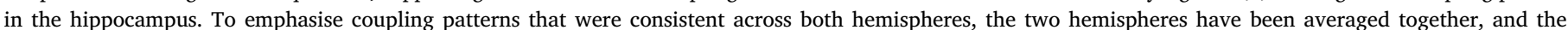

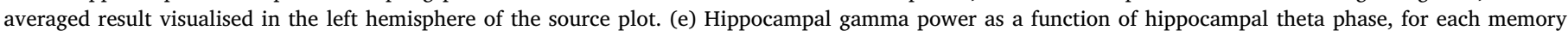

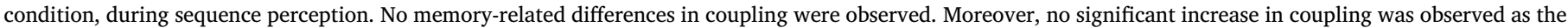

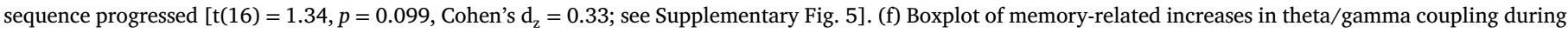

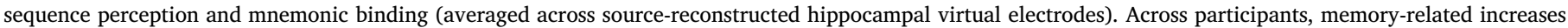

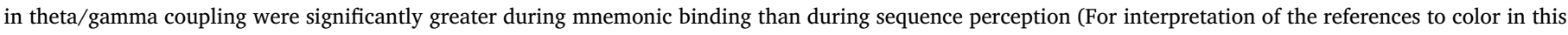
figure legend, the reader is referred to the web version of this article.).

tion. Specifically, could this be driven by independent changes in the theta oscillation or gamma oscillations? To address this, we contrasted the power of these oscillations between mnemonic binding and sequence perception, guided by the principle that a change in power would reflect a change in the driving oscillation. When contrasted the peak hippocampal theta, we saw significant reductions in power for sequence perception relative to mnemonic binding $(\mathrm{t}(16)=1.84, p=0.042$, Cohen's $\mathrm{d}_{\mathrm{z}}=0.45$ ). However, no difference was observed in the gamma band $\left(\mathrm{t}(16)=1.31, p=0.105\right.$, Cohen's $\left.\mathrm{d}_{\mathrm{z}}=0.32\right)$. This would suggest that the absence of theta/gamma phase-amplitude coupling during sequence perception is, in part, attributable to a diminished theta oscillation.

3.4. A double dissociation between the timing of memory-related decreases in neocortical alpha/beta power decreases and memory-related increases in hippocampal theta/gamma phase-amplitude coupling

Lastly, we formalised the interaction between memory-related neocortical alpha/beta power decreases and hippocampal theta-gamma phase-amplitude coupling. To this end, we conducted a $2 \times 2$ repeated measures ANOVA with encoding stage (perception vs. binding) and metric (alpha/beta power decreases vs. theta-gamma coupling) as factors. This revealed a significant interaction where memory-related al- pha/beta power decreases and memory-related theta-gamma coupling increases are dependent on the nature of the ongoing cognitive task $[\mathrm{F}(1,16)=9.14, p=0.008$, partial eta squared $=0.36]$. There was no main effect of cognitive task $[\mathrm{F}(1,16)=0.99, p=0.33$, partial eta squared $=0.06]$, nor metric $[\mathrm{F}(1,16)=0.20, p=0.50$, partial eta squared $=0.03$ ]. These results, in conjunction with those reported in the sections above, suggest that a double dissociation exists between neocortical alpha/beta power decreases and hippocampal theta-gamma phase-amplitude coupling, with the former being most pronounced during periods of information processing and the latter being most pronounced during periods of mnemonic binding.

\section{Discussion}

Reductions in neocortical alpha/beta power and enhancements in hippocampal theta-gamma phase-amplitude coupling are thought to play dissociable roles in the formation of episodic memories (Hanslmayr et al., 2016), with the former supporting information representation and the latter supporting mnemonic binding. As such, one would expect that these neural phenomena are temporally dissociable, with alpha/beta power decreases arising first (supporting the processing of incoming information) and theta-gamma phase-amplitude cou- 
pling arising later (supporting mnemonic binding, which can only arise after the information has initially been processed in the cortex). Here, we found just that. Memory-related decreases in neocortical alpha/beta power principally arose during sequence perception, while memoryrelated increases in hippocampal theta/gamma phase-amplitude coupling principally arose during a time window in which participants could mnemonically bind the sequence together. This double dissociation suggests that alpha/beta power decreases and hippocampal theta/gamma phase-amplitude coupling arise at two distinct stages in the memory formation process.

The representation of information relating to a to-be-encoded memory is thought to be supported by neocortical alpha/beta power decreases (Hanslmayr et al., 2012). Information theory proposes that unpredictable states, such as desynchronised neural networks, carry more information than predictable states (Shannon and Weaver, 1949). In line with this hypothesis, we found that memory-related reductions in alpha/beta power (an index for neural desynchrony) only arose when participants were required to process information about a sequence, and not when the sequence was being bound together (i.e., when no further information was presented for processing). The restriction of alpha/beta power decreases to time points where information can be processed adds further support to the idea that alpha/beta power decreases correlate with the representation of information relevant to episodic memories (Griffiths, et al., 2019a; Hanslmayr et al., 2012).

Alternatively, one could view this neocortical alpha activity as a correlate of attention (Jensen and Mazaheri, 2010; Klimesch et al., 2007). For example, when participants attend more intently to the stimuli, one might expect to observe both an alpha power decrease (e.g., Klimesch et al. 1998) and an increased likelihood of successful memory formation (Chun and Turk-Browne, 2007). Such an interpretation, however, is by no means adversarial to the idea that reductions in neocortical alpha/beta power reflect enhanced information representation. Indeed, as a participant attends more intently to a stimulus, one could expect that the stimulus is more clearly represented within the cortex. While we cannot disentangle these overlapping theories here, future studies could address this by asking whether an alpha/beta power decrease is predictive of information representation after controlling for the influence of attention.

During mnemonic binding, hippocampal theta-gamma phaseamplitude coupling scaled with memory performance. Mechanistically speaking, these increases may reflect a heightened degree of long-term potentiation (LTP) within the hippocampus. By coupling gamma oscillations resonating at a frequency optimal for spike-timing dependent plasticity (STDP; Bi and Poo, 1998; Nyhus and Curran, 2010) to the phase of theta optimal for LTP (Hasselmo et al., 2002), the potential for building synaptic connections between hippocampal neurons is increased greatly. Based on such ideas, one could speculate that the memoryrelated theta-gamma coupling observed during the binding window reflects the transformation of the three discrete sequence stimuli into a singular cohesive episodic memory.

In contrast, hippocampal theta-gamma phase-amplitude coupling during sequence perception did not relate to memory. This may be attributable to the diminished theta oscillation observed during sequence perception relative to that observed during mnemonic binding, which consequently may impair coupling between the theta and gamma bands. One could speculate that if the role of theta is to support mnemonic binding, then it may be naturally reduced during periods of the task where binding demands are less (e.g., during sequence perception). Going further, it may even be actively suppressed to avoid interfering with other task processes. Of course this is speculation, but these ideas could be tested by artificially perturbing theta activity during sequence perception and exploring its impact on behaviour.

If neocortical desynchrony and hippocampal synchrony are dissociable, does this mean that they act in complete independence of one another during encoding? Here, we would argue "no". Mnemonic binding cannot occur if the relevant information has not been perceived, as there is no information to bind. Therefore, one could expect that the underlying neural correlates of mnemonic binding are contingent on the prior neural processing of relevant information. In line with such ideas, prior work has shown that the magnitude of hippocampal gamma synchronisation can be predicted by preceding neocortical alpha/beta power decreases (Griffiths et al., 2019b; see Supplementary Fig. 7 for complementary findings within the data reported here). As such, one could speculate that neocortical desynchrony and hippocampal synchrony correlate with distinct cognitive processes (as evidenced above), but both neural phenomena (and the associated cognitive processes) must arise and interact to create an episodic memory.

We did not observe any memory-related fluctuations in theta power during the binding window. This is somewhat surprising; numerous previous studies have reported fluctuations in theta power correlating with later memory performance (for reviews, see Herweg et al. 2019 and Nyhus and Curran 2010). However, given that theories regarding theta and long-term potentiation (Bi and Poo, 1998; Hanslmayr et al., 2016; Nyhus and Curran, 2010) emphasise the importance of phase for LTP, rather than power, one could speculate that theta power has less to do with enhanced mnemonic binding, and as such, should not substantially correlate with successful memory formation. Similarly, we did not observe memory-related fluctuations in gamma power during mnemonic binding despite numerous studies demonstrating this previously (Burke et al., 2013; Griffiths et al., 2019b; Long and Kahana, 2015; Osipova et al., 2006). However, this can be explained by the fact that theta-gamma coupling was observed during this same window. If memory-related increases in gamma power are restricted to certain phases of theta, and theta is not stimulus-locked across trials, then across-trial averages of gamma power will sum to zero. As such, the absence of a 'pure' gamma effect here is not surprising.

It is worth noting that a growing amount of evidence suggests that ocular activity may be tied to the neural correlates of memory. For example, saccades that arise during the visual exploration of an object are known to induce a theta phase reset in the hippocampus (Jutras et al., 2013), while pupil dilation is known to correlate with cortical alpha/beta power following the presentation of a fear-conditioned stimulus (Dahl et al., 2020). As such, it is possible that ocular activity mediates our observed link between neocortical alpha/beta activity, hippocampal theta/gamma activity, and successful memory formation. Unfortunately, we did not acquire any measures of ocular activity and hence cannot address this idea here, but future studies may benefit from exploring the potential mediating effect of ocular activity on human memory formation.

In sum, these results demonstrate that decreases in neocortical alpha/beta power and increases in hippocampal theta/gamma phaseamplitude coupling are temporally dissociable in episodic memory formation (Hanslmayr et al., 2016).

\section{Credit authorship contribution statement}

Benjamin J. Griffiths: Conceptualization, Investigation, Formal analysis, Writing - original draft, Writing - review \& editing. María Carmen Martín-Buro: Formal analysis, Writing - review \& editing. Bernhard P. Staresina: Conceptualization, Writing - review \& editing, Supervision, Funding acquisition. Simon Hanslmayr: Conceptualization, Writing - original draft, Writing - review \& editing, Supervision, Funding acquisition.

\section{Code availability}

Code used to analyse this data is publicly available at: https://github. com/benjaminGriffiths/dissociating-encoding-oscillations

\section{Data availability}

Data is available at: https://openneuro.org/datasets/ds003694 


\section{Supplementary materials}

Supplementary material associated with this article can be found, in the online version, at doi:10.1016/j.neuroimage.2021.118454.

\section{References}

Aru, J., Priesemann, V., Wibral, M., Lana, L., Pipa, G., Singer, W., Vicente, R., 2014. Untangling cross-frequency coupling in neuroscience. Curr. Opin. Neurobiol. 31, 51-61. doi:10.1101/005926, September 2014.

Bahramisharif, A., Jensen, O., Jacobs, J., Lisman, J., 2018. Serial representation of items during working memory maintenance at letter-selective cortical sites. PLoS Biol., 171660 doi:10.1101/171660.

Bi, G., Poo, M., 1998. Synaptic modifications in cultured hippocampal neurons: dependence on spike timing, synaptic strength, and postsynaptic cell type. J. Neurosci. 18 (24), 1-9. doi:10.1038/25665.

Burke, J.F., Zaghloul, K.a., Jacobs, J., Williams, R.B., Sperling, M.R., Sharan, a.D., Kahana, M.J., 2013. Synchronous and asynchronous theta and gamma activity during episodic memory formation. J. Neurosci. 33 (1), 292-304. doi:10.1523/JNEUROSCI.2057-12.2013.

Buzsáki, G., Anastassiou, C.A., Koch, C., 2012. The origin of extracellular fields and currents-EEG, ECoG, LFP and spikes. Nat. Rev. Neurosci. 13 (6), 407-420. doi:10.1038/nrn3241.

Chun, M.M., Turk-Browne, N.B., 2007. Interactions between attention and memory. Curr. Opin. Neurobiol. 17 (2), 177-184. doi:10.1016/j.conb.2007.03.005.

Dahl, M., Mather, M., Sander, M., Werkle-Bergner, M., 2020. Noradrenergic responsiveness preserves selective attention across the adult lifespan. J. Neurosci. 40 (22), 43724390. doi:10.1101/551879.

Dalal, S., Jerbi, K., Bertrand, O., Adam, C., Ducorps, A., Schwartz, D., Martinerie, J., Lachaux, J.P., 2013. Simultaneous MEG-intracranial EEG: new insights into the ability of MEG to capture oscillatory modulations in the neocortex and the hippocampus. Epilepsy Behav. 28 (2), 283-302. doi:10.1016/j.yebeh.2013.03.012.

Fellner, M.C., Bäuml, K.H.T., Hanslmayr, S., 2013. Brain oscillatory subsequent memory effects differ in power and long-range synchronization between semantic and survival processing. NeuroImage 79, 361-370. doi:10.1016/j.neuroimage.2013.04.121.

Griffiths, B.J., Fuentemilla, L., 2019. Event conjunction: how the hippocampus integrates episodic memories across event boundaries. Hippocampus 1-15. doi:10.1002/hipo.23161.

Griffiths, B.J., Martín-Buro, M.C., Staresina, B.P., Hanslmayr, S., Staudigl, T., 2021. Al$\mathrm{pha}$ /beta power decreases during episodic memory formation predict the magnitude of alpha/beta power decreases during subsequent retrieval. Neuropsychologia 153 (2021), 107755. doi:10.1101/2020.07.08.193763.

Griffiths, B.J., Mayhew, S.D., Mullinger, K.J., Jorge, J., Charest, I., Wimber, M., Hanslmayr, S., 2019a. Alpha/beta power decreases track the fidelity of stimulusspecific information. ELife 8, 1-22. doi:10.32470/ccn.2019.1199-0, e49562.

Griffiths, B.J., Parish, G., Roux, F., Michelmann, S., Plas, M.Van Der, Kolibius, D., Chelvarajah, R., Rollings, D.T., Sawlani, V., Hamer, H., Gollwitzer, S., Kreiselmeyer, G., Staresina, B.P., Wimber, M., Hanslmayr, S., 2019b. Directional coupling of slow and fast hippocampal gamma with neocortical alpha/beta oscillations in human episodic memory. Proc. Natl. Acad. Sci. U.S.A. 1-9. doi:10.1073/pnas.1914180116.

Haller, M., Donoghue, T., Peterson, E., Varma, P., Sebastian, P., Gao, R., Noto, T., Knight, R.T., Shestyuk, A., Voytek, B., 2018. Parameterizing neural power spectra. BioRxiv, 299859 doi:10.1101/299859.

Hanslmayr, S., Matuschek, J., Fellner, M.C., 2014. Entrainment of prefrontal beta oscillations induces an endogenous echo and impairs memory formation. Curr. Biol. 24 (8), 904-909. doi:10.1016/j.cub.2014.03.007.

Hanslmayr, S., Staresina, B.P., Bowman, H., 2016. Oscillations and episodic memoryaddressing the synchronization/desynchronization conundrum. Trends Neurosci. 39 (1), 16-25. doi:10.1016/j.tins.2015.11.004.

Hanslmayr, S., Staudigl, T., 2014. How brain oscillations form memories-a processing based perspective on oscillatory subsequent memory effects. NeuroImage 85 (Pt 2), 648-655. doi:10.1016/j.neuroimage.2013.05.121.

Hanslmayr, S., Staudigl, T., Fellner, M.C., 2012. Oscillatory power decreases and long-term memory: the information via desynchronization hypothesis. Front. Hum. Neurosci. 6 (74), 1-12. doi:10.3389/fnhum.2012.00074.

Hasselmo, M.E., Bodelón, C., Wyble, B.P., 2002. A proposed function for hippocampal theta rhythm: separate phases of encoding and retrieval enhance reversal of prior learning. Neural Comput. 14 (4), 793-817. doi:10.1162/089976602317318965.

Herweg, N.A., Solomon, E.A., Kahana, M.J., 2019. Theta oscillations in human memory. Trends Cogn. Sci. 1-20. doi:10.1016/j.tics.2019.12.006.

Heusser, A.C., Poeppel, D., Ezzyat, Y., Davachi, L., 2016. Episodic sequence memory is supported by a theta-gamma phase code. Nat. Neurosci. 19. doi:10.1038/nn.4374, August.

Jensen, O., Mazaheri, A., 2010. Shaping functional architecture by oscillatory alpha activity: gating by inhibition. Front. Hum. Neurosci. 4, 1-8. doi:10.3389/fnhum.2010.00186, November.
Jutras, M.J., Fries, P., Buffalo, E.A., 2009. Gamma-band synchronization in the macaque hippocampus and memory formation. J. Neurosci. 29 (40), 12521-12531. doi:10.1523/JNEUROSCL.0640-09.2009.

Jutras, M.J., Fries, P., Buffalo, E.A., 2013. Oscillatory activity in the monkey hippocampus during visual exploration and memory formation. Proc. Natl. Acad. Sci. U. S. A. 110 (32), 13144-13149. doi:10.1073/pnas.1302351110.

Karlsson, A.E., Wehrspaun, C.C., Sander, M.C., 2020. Item recognition and lure discrimination in younger and older adults are supported by alpha/beta desynchronization. Neuropsychologia 148. doi:10.1101/2020.04.22.055764.

Klimesch, W., Doppelmayr, M., Russegger, H., Pachinger, T., Schwaiger, J., 1998. Induced alpha band power changes in the human EEG and attention. Neurosci. Lett. 244 (2), 73-76. doi:10.1016/S0304-3940(98)00122-0.

Klimesch, W., Sauseng, P., Hanslmayr, S., 2007. EEG alpha oscillations: The inhibition-timing hypothesis. Brain Res. Rev. 53 (1), 63-88. doi:10.1016/j.brainresrev.2006.06.003.

Kutner, M.H., Nachtsheim, C.J., Neter, J., 2004. Applied Linear Regression Models, 4th ed. McGraw-Hill Irwin.

Lakens, D., 2013. Calculating and reporting effect sizes to facilitate cumulative science: a practical primer for t-tests and ANOVAs. Front. Psychol. 4. doi:10.3389/fpsyg. 2013.00863, NOV.

Lisman, J.E., Jensen, O., 2013. The theta-gamma neural code. Neuron 77 (6), 1002-1016. doi:10.1016/j.neuron.2013.03.007.

Long, N.M., Kahana, M.J., 2015. Successful memory formation is driven by contextual encoding in the core memory network. NeuroImage 119, 332-337. doi:10.1016/j.neuroimage.2015.06.073.

Manning, J.R., Jacobs, J., Fried, I., Kahana, M.J., 2009. Broadband shifts in local field potential power spectra are correlated with single-neuron spiking in humans. J. Neurosci. 29 (43), 13613-13620. doi:10.1523/JNEUROSCI.2041-09.2009.

Maris, E., Oostenveld, R., 2007. Nonparametric statistical testing of EEG- and MEG-data. J. Neurosci. Methods 164 (1), 177-190. doi:10.1016/j.jneumeth.2007.03.024.

Martín-Buro, M.C., Wimber, M., Henson, R.N., Staresina, B.P., 2020. Alpha rhythms reveal when and where item and associative memories are retrieved. J. Neurosci. 40 (12), 2510-2518. doi:10.1523/JNEUROSCI.1982-19.2020.

Murthy, V.N., Fetz, E.E., 1996. Oscillatory activity in sensorimotor cortex of awake monkeys: synchronization of local field potentials and relation to behavior. J. Neurophysiol. 76 (6), 3949-3967. doi:10.1152/jn.1996.76.6.3949.

Nyhus, E., Curran, T., 2010. Functional role of gamma and theta oscillations in episodic memory. Neurosci. Biobehav. Rev. 34 (7), 1023-1035. doi:10.1016/j.neubiorev.2009.12.014.

O’Neill, G.C., Barry, D.N., Tierney, T.M., Mellor, S., Maguire, E.A., Barnes, G.R., 2021. Testing covariance models for MEG source reconstruction of hippocampal activity. BioRxiv 1-19.

Oostenveld, R., Fries, P., Maris, E., Schoffelen, J.-M., 2011. FieldTrip: open source software for advanced analysis of MEG, EEG, and invasive electrophysiological data. Comput. Intell. Neurosci. 2011, 1-9. doi:10.1155/2011/156869.

Osipova, D., Takashima, A., Oostenveld, R., Fernandez, G., Maris, E., Jensen, O., 2006. Theta and gamma oscillations predict encoding and retrieval of declarative memory. J. Neurosci. 26 (28), 7523-7531. doi:10.1523/JNEUROSCI.1948-06.2006.

Parish, G., Hanslmayr, S., Bowman, H., 2018. The sync/desync model: how a synchronized hippocampus and a desynchronized neocortex code memories. J. Neurosci. 38 (14), 3428-3440. doi:10.1523/JNEUROSCI.2561-17.2018.

Quiroga, R.Q., Reddy, L., Kreiman, G., Koch, C., Fried, I., 2005. Invariant visual representation by single neurons in the human brain. Nature 435 (7045), 1102-1107. doi:10.1038/nature03687.

Ruzich, E., Crespo-García, M., Dalal, S.S., Schneiderman, J.F., 2019. Characterizing hippocampal dynamics with MEG: a systematic review and evidence-based guidelines. Hum. Brain Mapp. 40 (4), 1353-1375. doi:10.1002/hbm.24445.

Sederberg, P.B., Schulze-Bonhage, A., Madsen, J.R., Bromfield, E.B., McCarthy, D.C., Brandt, A., Tully, M.S., Kahana, M.J., 2007. Hippocampal and neocortical gamma oscillations predict memory formation in humans. Cereb. Cortex 17 (5), 1190-1196. doi:10.1093/cercor/bhl030.

Shannon, C.E., Weaver, W., 1949. A Mathematical Theory of Communication. University of Illinois Press.

Staudigl, T., Hanslmayr, S., 2013. Theta oscillations at encoding mediate the context-dependent nature of human episodic memory. Curr. Biol. 23 (12), 1101-1106.

Tort, A.B.L., Komorowski, R., Eichenbaum, H., Kopell, N., 2010. Measuring phaseamplitude coupling between neuronal oscillations of different frequencies. J. Neurophysiol. 104 (2), 1195-1210. doi:10.1152/jn.00106.2010.

Tort, A.B.L., Komorowski, R.W., Manns, J.R., Kopell, N.J., Eichenbaum, H., 2009. Thetagamma coupling increases during the learning of item-context associations. Proc. Natl. Acad. Sci. U. S. A. 106 (49), 20942-20947. doi:10.1073/pnas.0911331106.

Tulving, E., 2002. Episodic memory: from mind to brain. Annu. Rev. Psychol. 53, 1-25. doi:10.1146/annurev.psych.53.100901.135114.

van Veen, B., van Drongelen, W., Yuchtman, M., Suzuki, A., 1997. Localization of brain electrical activity via linearly constrained minimum variance spatial filtering. IEEE Trans. Biomed. Eng. 44 (9), 867-880. doi:10.1109/10.623056.

Zhang, H., Jacobs, J., 2015. Traveling theta waves in the human hippocampus. J. Neurosci. 35 (36), 12477-12487. doi:10.1523/JNEUROSCI.5102-14.2015. 